\title{
Gelbe Flecken und wie weiter?
}

Vadasz, V ; Fehr, J ; Gerth-Kahlert, C

DOI: https://doi.org/10.1007/s00347-014-3105-1

Other titles: Yellow stains and what now?

Posted at the Zurich Open Repository and Archive, University of Zurich ZORA URL: https://doi.org/10.5167/uzh-103285

Journal Article

Published Version

Originally published at:

Vadasz, V; Fehr, J; Gerth-Kahlert, C (2014). Gelbe Flecken und wie weiter? Der Ophthalmologe, 111(12):1213-1216.

DOI: https://doi.org/10.1007/s00347-014-3105-1 
Ophthalmologe 2014 · 111:1213-1216

DOI 10.1007/s00347-014-3105-1

Online publiziert: 6. Dezember 2014

(c) Springer-Verlag Berlin Heidelberg 2014

\section{Vadasz ${ }^{1} \cdot$ J. Fehr ${ }^{2} \cdot$ C. Gerth-Kahlert ${ }^{1}$}

${ }^{1}$ Augenklinik, UniversitätsSpital Zürich

${ }^{2}$ Klinik für Infektionskrankheiten und Spitalhygiene, UniversitätsSpital Zürich

\section{Gelbe Flecken und wie weiter?}

\section{Anamnese}

Ein 52-jähriger Patient wurde aufgrund einer subjektiv progredienten Sehverschlechterung beidseits zur Evaluierung des ophthalmologischen Status zugewiesen. Der Patient wurde zuvor wegen seit 2 Wochen bestehenden starken Kopfschmerzen mit anhaltender Emesis und Fieber in der Klinik für Infektionskrankheiten bei vorbekannter HIV-Infektion (CDC-Stadium C3) stationär aufgenommen. Zusätzlich war eine chronisch aktive Hepatitis-B-Infektion bekannt.

Zweieinhalb Wochen zuvor war eine hochaktive antiretrovirale Therapie (Etravirin, Raltegravir, Tenofivir, Lamivudin und Zidovudin) bei stark erniedrigter CD4-Zahl (13/ $\mu \mathrm{l})$ und einer Viruslast von HIV-RNA 47.200 Kopien/ml begonnen worden. Ein fraglicher Gewichtsverlust von $1-2 \mathrm{~kg}$ in den letzten Wochen wurde angegeben. Nachtschweiß bestand nicht.

Die neurologische Untersuchung einschließlich Schädel-MRT und -CT war unauffällig.

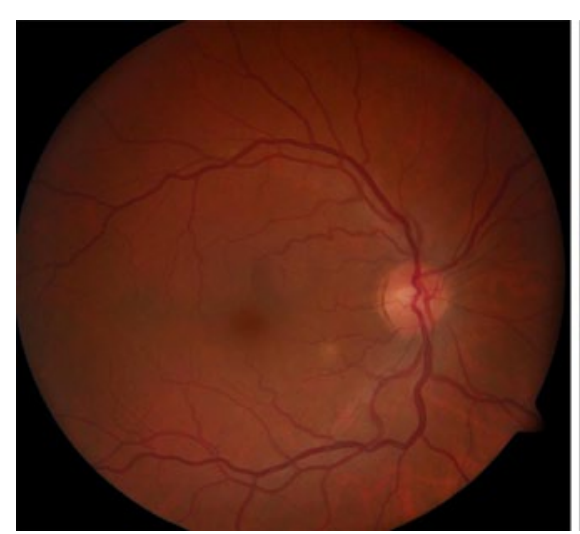

Abb. $1 \Delta$ Fundusfoto beidseits

\section{Klinischer Befund}

Die ophthalmologische Untersuchung erbrachte folgende Befunde: Fernvisus sc $0,8 \mathrm{p}$ beidseits, links mit $-0,75 / 65^{\circ}$ auf 1,0 p korrigierbar, Nahvisus mit $+1,5$ beidseits 1,0, Pupillen rund, isokor, mit regelrechter Pupillomotorik. Die Spaltlampenuntersuchung zeigte beidseits reizfreie und altersentsprechende vordere Augenabschnitte. Funduskopisch fielen rechts am inferotemporalen Gefäßbogen und links am superotemporalen Gefäßbogen kleine, gelbliche, wenig erhabene, scharf begrenzte Einlagerungen auf ( $\bullet$ Abb. 1). Eine Gefäßeinscheidung oder Blutungen bestanden nicht. Die Papille und Makula waren beidseits unauffällig. Zellen im Glaskörper waren nicht vorhanden.

\section{Weitere Befunde}

\section{Optische Kohärenztomographie}

In der optischen Kohärenztomographie (OCT)-Untersuchung sind korrelierend

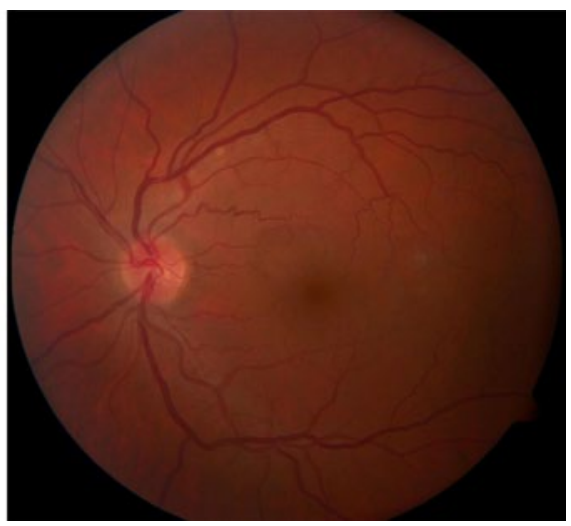




\section{Bild und Fall}
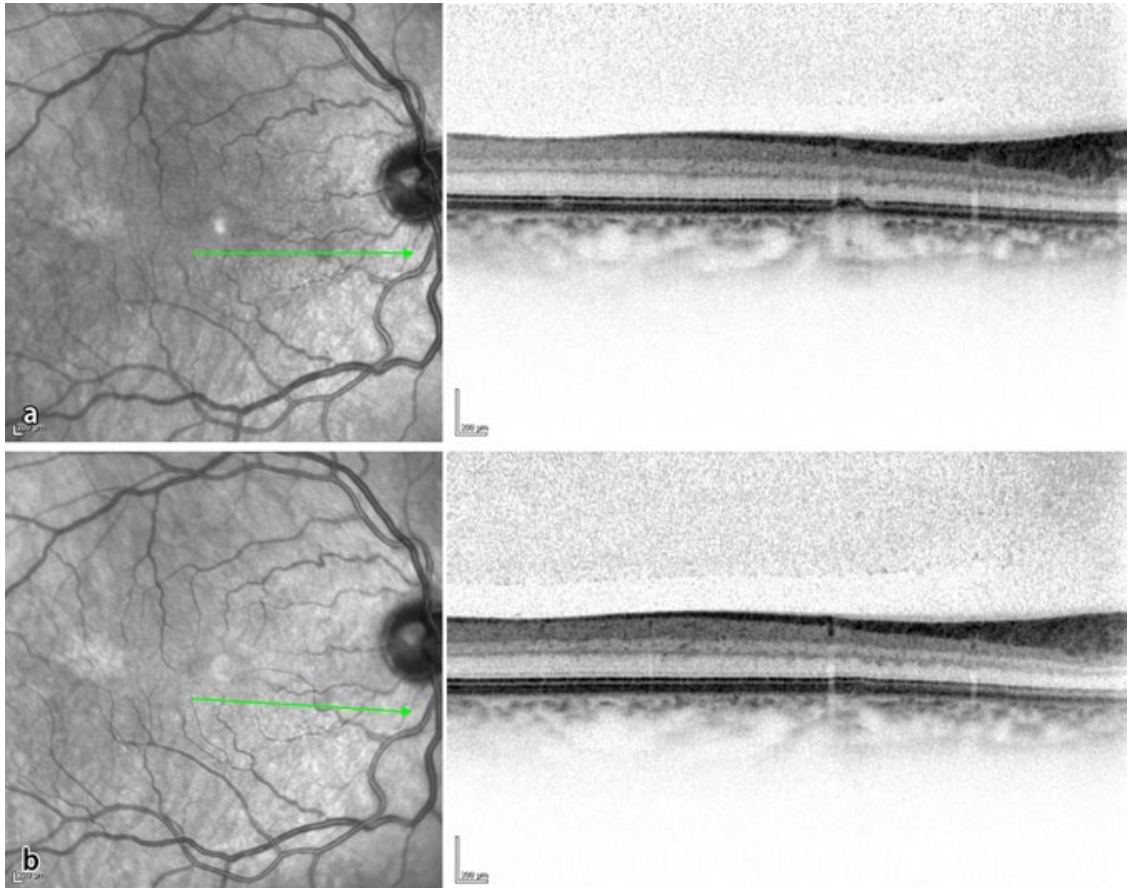

Abb. $2 \Delta$ Optische Kohärenztomographie - horizontaler Scan durch die Fundusläsion: a initial, b nach 11 Monaten
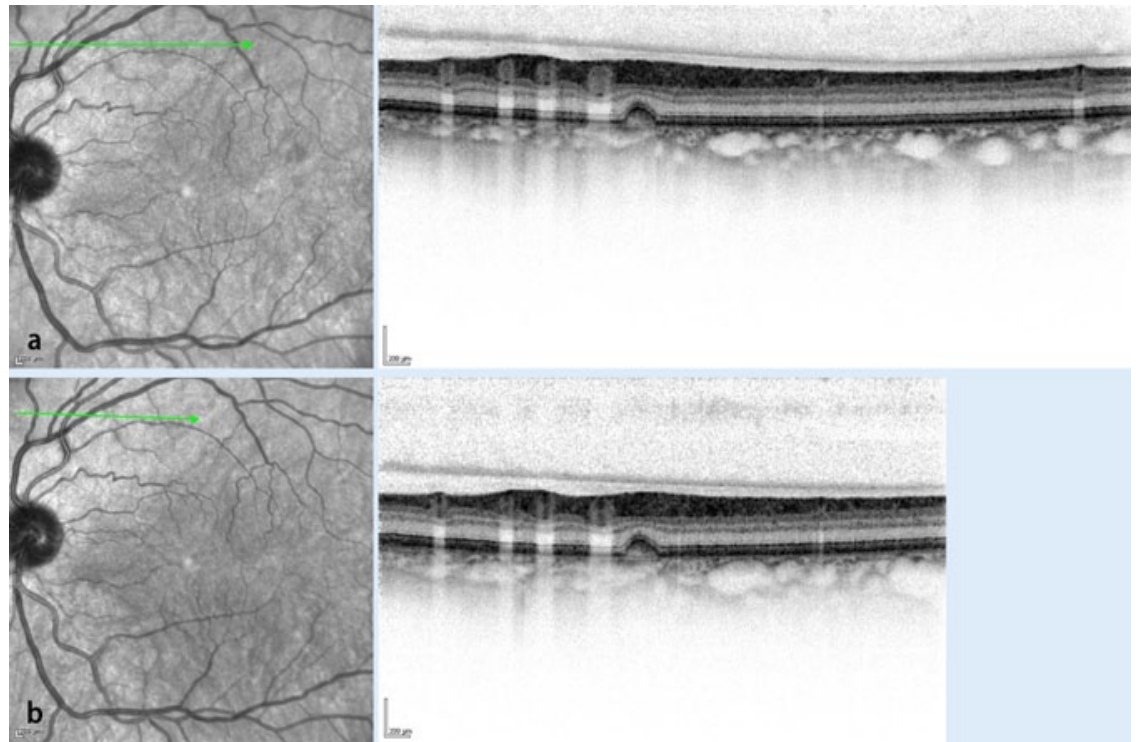

Abb. $3 \Delta$ Optische Kohärenztomographie - horizontaler Scan durch die Fundusläsion: a initial, b nach 11 Monaten

zu den gelblichen Fundusläsionen Unterbrechungen der choriokapillaren Struktur am rechten Auge darstellbar. Im Bereich der äußeren Netzhautschichten ist eine fokale Unterbrechung mit zusätzlich flacher Abhebung des retinalen Pigmentepithelbandes zu sehen (• Abb. 2). Am linken Auge zeigt sich korrelierend zu den gelblichen Läsionen eine drusenoide Pigmentepithelabhebung mit dar- wiegend hypofluoreszent, teilweise auch hyperfluoreszent mit Staining. In der Indocyaningrün (ICG)-AngiographieUntersuchung stellen sich die Läsionen teils hyper- teils hypofluoreszent dar (• Abb.4). unter liegendem hyperreflexivem, granulärem Material (• Abb. 3). Beidseits ist keine sub- und intraretinale Flüssigkeitsansammlung sichtbar.

\section{Fluoreszenz- und Indocyaningrün-Angiographie}

In der Fluoreszenzangiographie (FAG) zeigen sich die gelblichen Läsionen vor- 

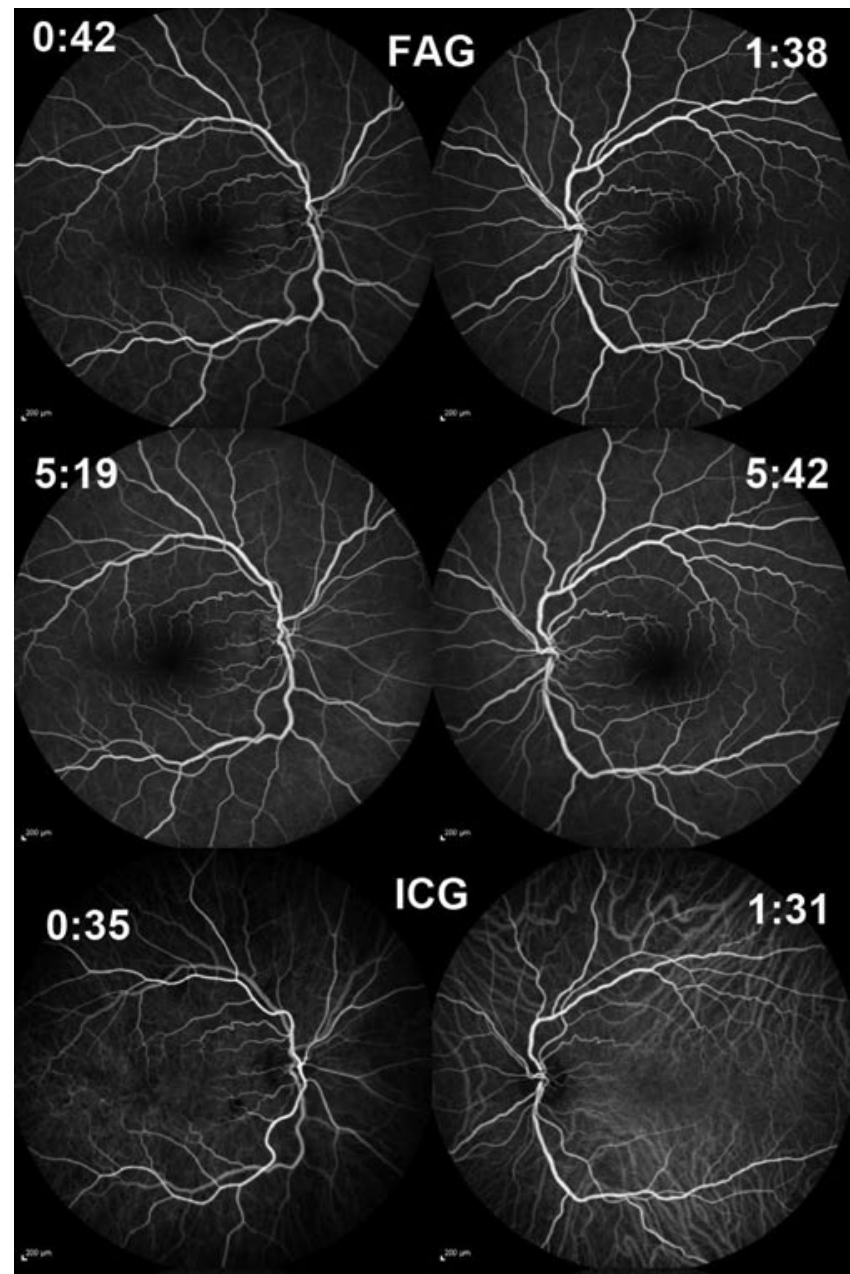

$10: 31$

11:01

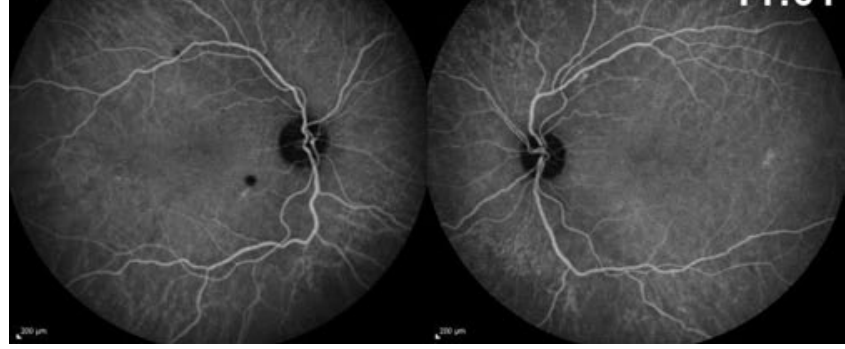

Abb. $4<$ Fluoreszenz-

angiographie (FAG)

und Indocyaningrün

(ICG)-Angiographie

\section{Wie lautet Ihre Diagnose?}

》) Diagnose: Kryptokokkokenmeningoenzephalitis mit chorioretinaler Beteiligung

\section{Therapie}

Es wurde eine antimykotische Therapie mit Flucytosin und Amphotericin B eingeleitet, die jedoch nach 13 Tagen aufgrund einer akuten Niereninsuffizienz gestoppt werden musste. Es erfolgte eine Umstellung auf Fluconazol per os. Während des stationären Aufenthaltes wurde bei unklaren Fieberschüben mittels Polymerasekettenreaktion eine Zytomegalievirusinfektion ohne Organmanifestationen diagnostiziert und mit Ganciclovir therapiert.

\section{Verlauf}

Unter oben genannter Therapie zeigte sich der Patient im Verlauf beschwerdefrei. In der abschließenden ophthalmologischen Kontrolle nach 3 Monaten zeigten sich bei vollem Visus die Fundusveränderungen deutlich regredient und konnten nur noch ansatzweise erkannt werden. 


\section{Hintergrundinformationen}

Cryptococcus neoformans ist ein ubiquitär vorkommender, bekapselter Pilz, der durch Inhalation und hämatogene Streuung die Endorgane mit Affinität zum zentralen Nervensystem erreicht. Weitere Manifestationsorte sind die Haut, die Knochen sowie die Leber [1]. Eine Infektion ist bei intaktem Immunsystem eine absolute Rarität. Bei immungeschwächten Personen stellt jedoch die Kryptokokkokenmeningoenzephalitis eine häufige Krankheitsmanifestation dar. Eine okuläre Beteiligung kann unter anderem bei ausgeprägter Meningitis mit Sepsis, malignen Erkrankungen, systemischem Lupus erythematodes, fortgeschrittener HIV-Infektion und weiteren Immunschwächekrankheiten auftreten. Das Auge wird entweder durch direkte Infizierung über den N. opticus oder durch hämatogene Streuung erreicht [1]. Primär zeigen sich Veränderungen an der Choroidea, die klinisch als diskrete, gelblich-weißliche, leicht erhabene Läsionen sichtbar werden [1]. In der Literatur werden zudem eine retinale Nekrose mit begleitender retinaler Hämorrhagie sowie exsudative Netzhautablösungen beschrieben [2]. Das Papillenödem wird als häufigste Auffälligkeit bei okulärer Beteiligung der Kryptokokkokenmeningoenzephalitis beschrieben. Diese wird mit dem dabei mehrheitlich erhöhten intrakraniellen Druck in Zusammenhang gestellt [3]. Andere Augenmanifestationen umfassen folgende Erkrankungen: Konjunktivitis, Iritis, Choroiditis, Chorioretinitis, Vitritis und Endophthalmitis [4].

\section{Diagnostik}

Die ophthalmologische Diagnostik basiert auf der klinischen Charakteristik einschließlich der FAG, die typischerweise multifokale, rundliche, hypofluoreszente Veränderungen ohne Leckage zeigt. Die Diagnosesicherung erfolgt im interdisziplinären Team. Entscheidend sind ein erhöhter Liquoreröffnungsdruck und der Nachweis des Kryptokokkenantigens im Liquor und im Serum. Weiter kann neben einer India-Ink-Färbung eine Pilzkultur herangezogen werden.

\section{Allgemeine Therapie}

Die initiale Therapie besteht aus Amphotericin B und Flucytosine. Zur Erhaltungstherapie stehen Voriconazol sowie Fluconazol mit jeweils hoher Bioverfügbarkeit und hohen erreichten intravitrealen Konzentrationen zur Verfügung. Eine frühe Pars-plana-Vitrektomie sollte nur bei schwerer, therapieresistenter Vitritis durchgeführt werden [5].

\section{Fazit für die Praxis}

- Eine multifokale, infektiöse Choroiditis kann auf eine disseminierte Kryptokokkose hinweisen, die ohne adäquate Therapie eine infauste Prognose hat.

- Bei der ophthalmologischen Betreuung von immungeschwächten $\mathrm{Pa}$ tienten, wie beispielsweise einer fortgeschrittenen HIV-Infektion, bedürfen multifokale Fundusläsionen immer einer weiterführenden und detaillierten Beurteilung, um eine disseminierte Kryptokokkeninfektion auszuschließen.

\section{Korrespondenzadresse

Dr.V.Vadasz
Augenklinik,
UniversitätsSpital Zürich
Frauenklinikstr. 24,8091 Zürich
Schweiz
vinzenz.vadasz@usz.ch

Danksagung. Wir bedanken uns bei der Fotoabteilung für die Aufarbeitung der Fundus- und Angiographiebilder sowie bei Frau PD Dr. med. S. Zweifel für wertvolle Hinweise.

Interessenkonflikt. V. Vadasz, J. Fehr und C. GerthKahlert geben an, dass kein Interessenkonflikt besteht.

\section{Literatur}

1. Arévalo JF (2013) Retinal and choroidal manifestations of selected systemic diseases, 1. Aufl. Springer, Berlin Heidelberg New York Tokyo, S 539

2. Shulman J, Cruz EL de la, Latkany P et al (2009) Cryptococcal chorioretinitis with immune reconstitution inflammatory syndrome. Ocul Immunol Inflamm 17(5):314-315

3. Kestelyn P, Taelman H, Bogaerts J et al (1993) Ophthalmic manifestations of infections with Cryptococcus neoformans in patients with the acquired immunodeficiency syndrome. Am J Ophthalmol 116(6):721-727
4. Wykoff CC, Albini TA, Couvillion SS et al (2009) Intraocular cryptococcoma. Arch Ophthalmol 127(5):700-702

5. Henderly DE, Liggett PE, Rao NA (1987) Cryptococcal chorioretinitis and endophthalmitis. Retina 7(2):75-79 